\title{
EFFECT OF FRETTING-WEAR ON DYNAMIC ANALYSIS. COMPARISON BETWEEN EXPERIMENTAL RESULTS AND NUMERICAL SIMULATIONS FOR A VIBRATORY FRICTION RIG.
}

\author{
F. TUBITA*, A. FANTETTI ${ }^{\dagger}$, L. BLANC* and F.THOUVEREZ* \\ * École Centrale de Lyon, CNRS UMR 5513 \\ LTDS, F-69134 Ecully Cedex, France \\ e-mail: fabia.tubita@ec-lyon.fr, web page: https://www.ec-lyon.fr/ \\ † Imperial College London, Exhibition Rd, London, UK
}

Key words: Non-linear Forced Response, Friction Contact, Fretting-Wear, Multi-scale Problems

\begin{abstract}
Dry friction in contact interfaces can have an important impact on the dynamic response of jointed structures subjected to vibration. It may cause frettingwear leading to a modification of the contact surface geometry by producing wear debris through material removal and dissipating energy. Consequently, the contact behaviour is modified and the worn geometry induces a change in vibrations level. Therefore, it is important to be able to simulate these complex phenomena occurring at the interfaces to predict the forced response of assembled structures and also their life-expectancy to design high confidence components. A multi-scale approach is implemented considering a slow-scale for wear phenomena and a fast-scale for the non-linear dynamic response and applied to validate an experimental test.
\end{abstract}

\section{INTRODUCTION}

Fretting-wear appears between two contacting surfaces, subjected to oscillatory relative movement of small amplitude. The evolution of wear occurs over a long period, on a micro-scale level, but may impact the dynamic response of assembled structures. Contact surfaces between root-blade and slot-disk, friction dampers under platform, blade dovetail or fir-tree slots on assembled rotors, shroud contacts and bolted flange joints are the typical industrial applications in which fretting occurs. The importance to predict the vibration response of mechanical assemblies in the presence of this phenomenon is relevant to estimate the performance as well as the lifetime of every component, under performance, in terms of damping and also when resonant frequencies change with 
wear. Several numerical [1]-[2] and experimental [3]-[4] approaches have been developed to calculate the vibrational response in presence of friction. Salles et Al. [5]-[6] proposed a multi-scale approach, based on the Dynamic Lagrangian Frequency-Time scheme [2] and on the coupling between dynamic (fast time scale) and tribological (slow time scale) phenomena. Various methods have also been proposed to evaluate wear, based on the Harmonic Balance Method (HBM), consisting of a Galerkin's procedure with Fourier basis, coupled with a semi-analytical contact solver [7]-[8]-[9], to consider finer contact surface discretizations. In previous studies, [5]-[6] academic examples are proposed to describe the phenomenon. In this study, an application of the improved DLFT method increasing wear depth along time [6] is applied to the experimental test rig [10], detailed in the next section. The test rig dynamics reproduces the dynamic behaviour observed in friction dampers and may give a detailed frictional description of contacting interfaces, to allow more accurate modelling of assembled structures. The added value is mainly in the comparison between experiments and updated simulations. This enables 1) to improve the understanding of phenomena at work, reproducing numerically the physics of the problem to predict the worn geometry and the wear volume along time; 2) to validate the optimal number of elements required to reach targeted level of fidelity in predictions

at reasonable costs; 3 ) to access elements of scenario that are under the scope of exper-

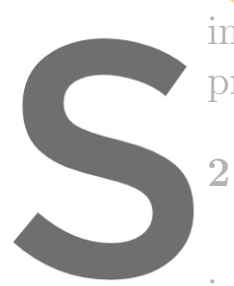
iments, because intrusive sensors prevent
propose information at this level.
2 EXPERIMENTAL SETUP
The experimental benchnark used in th

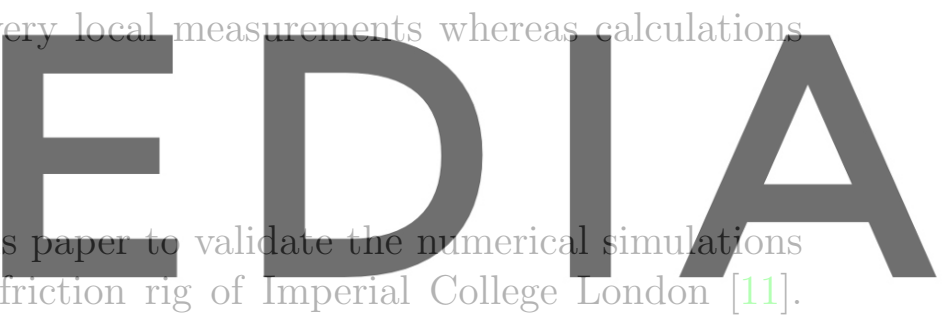

is a set of experiments performed on the friction rig of Imperial College London

Register for free at httos yas built to provide input contact parameters for non linear dynamic analysis by measuring high-1requency hysteresis loops. From the hysteresis loops, friction coefficient and tangential contact stiffness are extracted. A set of experiments from this friction rig was published in [10] and used here to validate the simulations of the proposed numerical method.

\subsection{Description of the test rig}

The friction rig generates a flat-on-flat sliding contact between a pair of cylindrical specimens as illustrated in Figure 1a. The rig is composed of a moving block that slides over a static block. The moving block is excited using a shaker and is composed of a moving arm hinged to a larger moving mass. One specimen is clamped to the moving arm and is in contact with the other specimen that is clamped to the static block. The photo of the rig is shown in Figure 1b. The relative displacement between the sliding specimens is measured slightly above and below the contact interfaces, less than $1 \mathrm{~mm}$ far from the contact, using two Laser Doppler vibrometers. This accurate measurement method leads to a negligible effect on the bulk elastic deformation of the specimens, 
making the measurement of the tangential contact stiffness more reliable. The friction force is measured with dynamic load cells attached to the static block. A continuous contact is ensured between the specimens by applying a normal load by means of a pneumatic actuator placed on the top of the moving block.
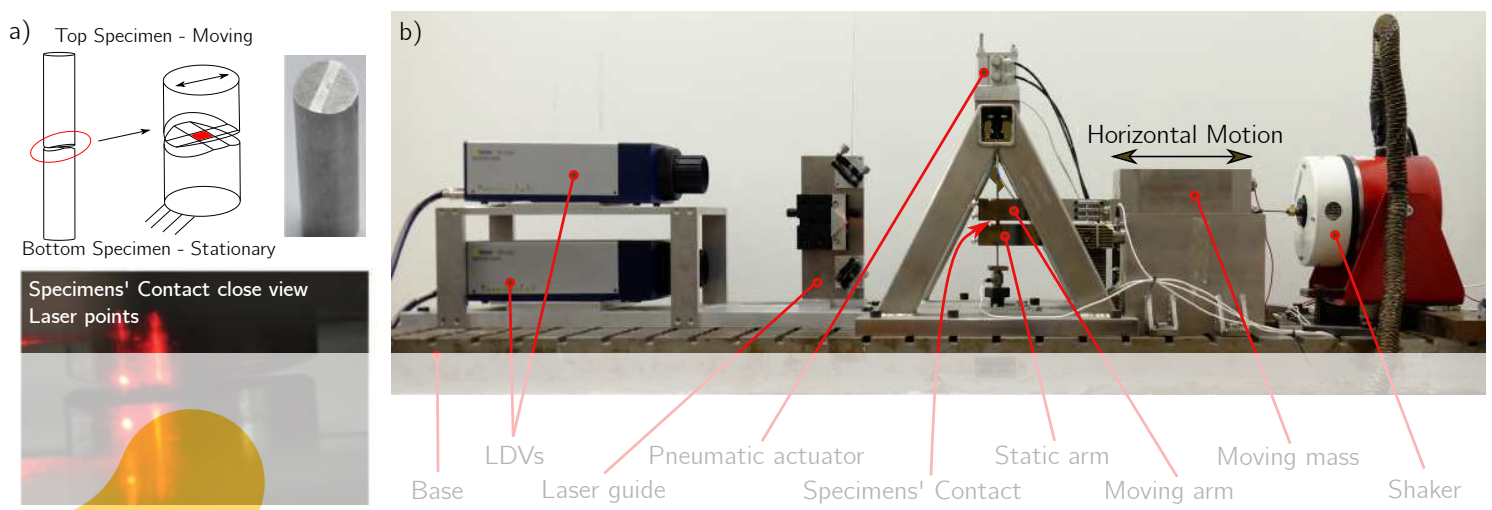

Figure 1: a) Specimen arrangement for the flat-on-flat contact [10]; b) Photo of the friction rig

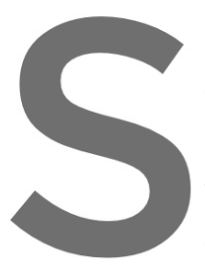

A typical measurec
obtained by dividing
normal load, and the
the loop. In a previous
over different time spa
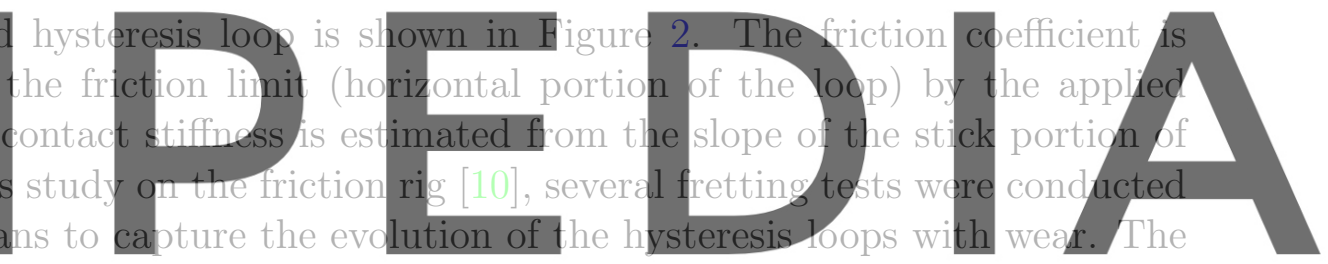

experimental plan of that study is described in the following paragraph and its results are

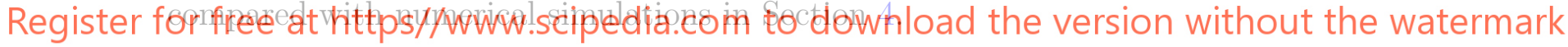

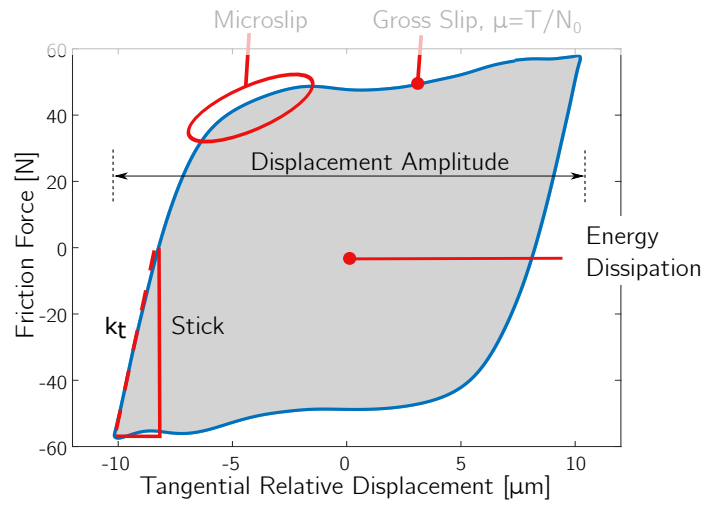

Figure 2: Typical measured hysteresis loop from the friction rig [10] 


\subsection{Wear test plan}

A series of five fretting tests was conducted using different couples of specimens at room temperature.[10] The excitation frequency was $100 \mathrm{~Hz}$, which is the best working frequency for the test rig. This frequency is of interest because in the middle of the first mode, which is highly non-linear [11]. The normal load was maintained constant at 60 $\mathrm{N}$ for all tests. These values were chosen to generate hysteresis loops with the necessary amount of energy dissipated and, therefore, of fretting wear. The specimens were all made of stainless steel (SS304) and their contact interface was carefully hand polished leading to a roughness value Ra of about $0.1 \mu \mathrm{m}$. The width of the contact was maintained at $1 \mathrm{~mm}$ on each specimen. In every test, the specimens were placed orthogonally as illustrated in Figure 1a and for each pair the nominal area of contact was $1 \mathrm{~mm}^{2}$ (having a $5 \%$ of variability due to the manufacturing tolerance and assembly). The resulting nominal contact pressure was therefore $60 \mathrm{MPa}$. Experiments were carried out at imposed lateral force amplitude. Two tangential excitation amplitudes of $53 \mathrm{~N}$ and $75 \mathrm{~N}$ were chosen to investigate the effect of different strokes, on average respectively $14 \mu \mathrm{m}$ and $22 \mu \mathrm{m}$ of full relative sliding. Two tests were conducted at $53 \mathrm{~N}$ excitation (average $14 \mu \mathrm{m}$ sliding) for about $5.5 \mathrm{~h}$ each, and three tests were conducted at $75 \mathrm{~N}$ excitation (average $22 \mu \mathrm{m}$ sliding) for 0.6, 3.6 and 9 h respectively to assess the repeatability of the experiments.

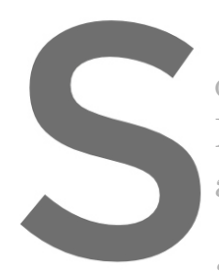

The numerical mol

ditions on the test

N excitation, average

analysis.

3 NUMERICAL METHOD
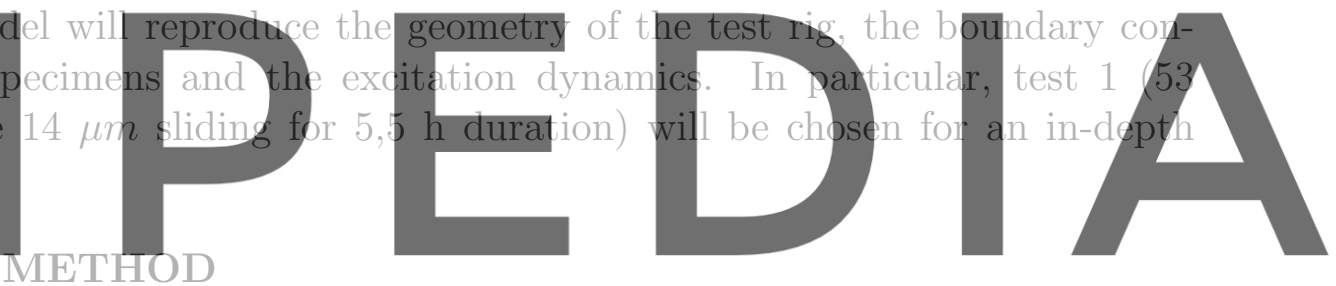

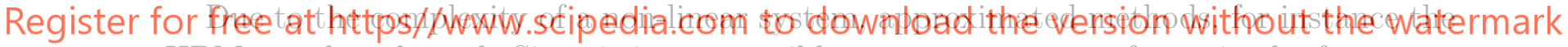

HBM, can be adopted. Since it is not possible to express contact forces in the frequency domain, an alternating frequency-time (AFT) procedure [14] is performed to estimate them in the time domain. In this paper, the non-linear forced response is evaluated using the HBM coupled with the Dynamics Lagrangian Frequency-Time scheme (DLFT) developed by Nacivet et al. [2]. The contact zone is defined by a node-to-node contact element and for each contact element, this method takes into account directly the Coulomb friction's law, without any softening, and the non-interpenetration condition to compute the contact forces in the time domain.

Wear is calculated at each interface node considering Archard's formulation [12] assuming that the friction coefficient is not affected by the wear evolution. The roughness is here geometrically neglected. It is considered that the depth of wear is very small relatively to the characteristic dimensions of the structures in contact, which is why the modifications due to wear of the mass and stiffness matrices are neglected. 


\subsection{Frequency-domain formulation}

The equations of motion obtained after discretization for two solids $(i=1,2)$ in contact with friction are given as follow:

$$
M^{i} \ddot{U}^{i}+C^{i} \dot{U}^{i}+K^{i} U^{i}+F_{c}^{i}\left(U^{i}, \dot{U}^{i}, W^{i}\right)=F_{e x}^{i}
$$

where:

- $\boldsymbol{M}^{i}, \boldsymbol{C}^{i}$ and $\boldsymbol{K}^{i}$ are the mass, damping and stiffness matrices, respectively, for each body $i . \quad \boldsymbol{M}^{i}$ and $\boldsymbol{K}^{i}$ are extracted from the FE models for both of them. $\boldsymbol{C}^{i}$ is introduced by means of a Rayleigh's damping matrix. To reduce the size of the system a Craig Bampton-Component Mode Synthesis (CB-CMS) [13] reduction is applied;

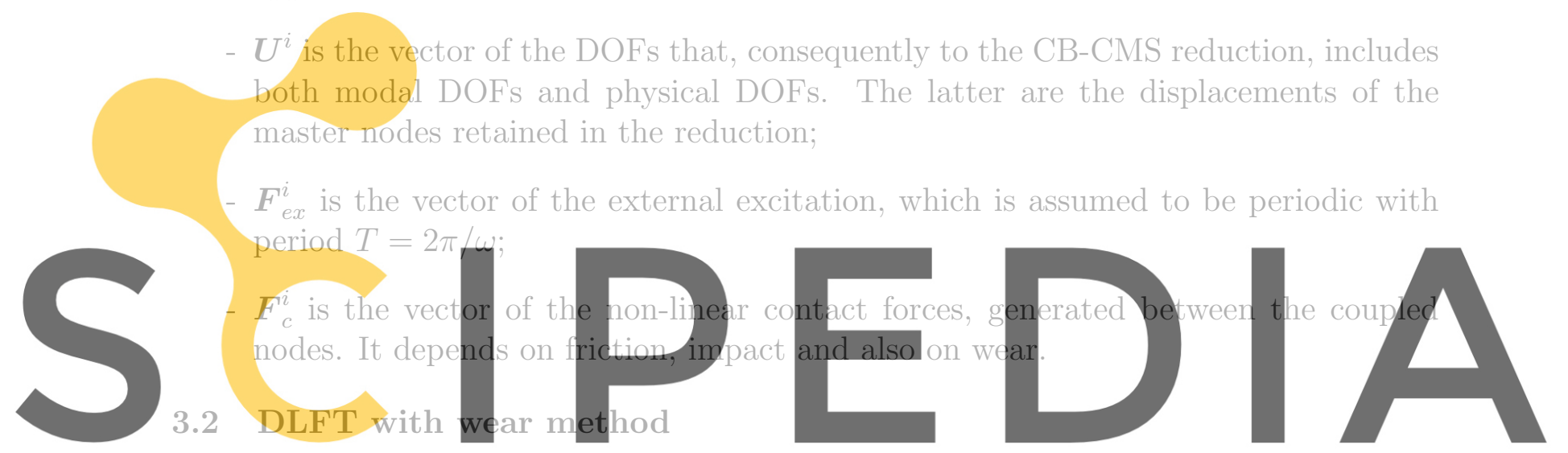

The complete and reduced solutions of Eq. (1) are studied in the framework of a

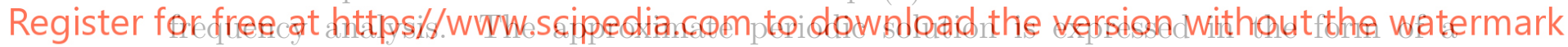

truncated Fourier series. Here, two time scales are considered to couple dynamics and wear phenomena:

$$
\boldsymbol{U}(\tau, \eta)=\tilde{\boldsymbol{U}}_{\mathbf{0}}(\eta)+\sum_{i=1}^{N_{h}}\left(\tilde{\boldsymbol{U}}_{\boldsymbol{n}, \boldsymbol{c}}(\eta) \cos (i \tau)+\tilde{\boldsymbol{U}}_{\boldsymbol{n}, \boldsymbol{s}}(\eta) \sin (i \tau)\right)
$$

where $\tau$ represents the "fast" time scale, related to the vibrations and $\eta$ the "slow" time scale, related to the wear. To compute the periodic steady-state response in a frequency domain, a Galerkin's procedure is used. In addition, in frequency domain, the size of the problem can be reduced by performing two exact reductions [2]. The first one is a condensation on the interface DOFs (non-linear DOFs) and a further factor two reduction can be obtained by considering the problem in term of relative displacements. Eq. (1) is turned into a set of nonlinear algebraic equations:

$$
Z_{r} \tilde{U}_{r}(\eta)+\tilde{\lambda}(\eta)=\tilde{F}_{r}
$$


where $\tilde{\boldsymbol{U}}_{\boldsymbol{r}}$ and $\tilde{\boldsymbol{F}}_{\boldsymbol{r}}$ are respectively the multi-harmonic vectors of the relative displacements and reduced external forces. $\boldsymbol{\lambda}(\boldsymbol{\eta})$ is the vector of the Lagrange multipliers which represents the unknown contact forces vector in the frequency domain. $\boldsymbol{Z}_{\boldsymbol{r}}$ is the reduced relative stiffness matrix defined as:

$$
\boldsymbol{Z}_{r}=\operatorname{diag}\left(\mathbf{2} K, \boldsymbol{Z}_{k=1, \ldots, N_{h}}\right), \text { with } \quad \boldsymbol{Z}_{k}=\left[\begin{array}{cc}
\boldsymbol{K}-\left(k \omega^{2}\right) \boldsymbol{M} & k \omega \boldsymbol{C} \\
k \omega \boldsymbol{C} & \boldsymbol{K}-\left(k \omega^{2}\right) \boldsymbol{M}
\end{array}\right],
$$

A non-linear solver is then used to find the zero of the following function:

$$
f\left(\tilde{U}_{\boldsymbol{r}}\right)=Z_{\boldsymbol{r}} \tilde{U}_{\boldsymbol{r}}(\boldsymbol{\eta})+\tilde{\boldsymbol{\lambda}}(\boldsymbol{\eta})-\tilde{\boldsymbol{F}}_{\boldsymbol{r}}
$$

In this study, the Jacobian matrix used to compute Eq. (5) is evaluated semi-analytically as indicated in [6], obtaining a significant reduction in the computational time.

\subsection{Modeling of the contact forces}

Solving the previous algebraic system (3) requires a prior computation of the non-linear forces $\tilde{\boldsymbol{\lambda}}$. Unfortunately, it is not possible to calculate them directly in the frequency domain. An AFT scheme [14] is applied. It typically consists of evaluating displacements and velocities in the time domain, through a forward DFT, and then going back to the frequency domain, usin
Nacivet [2] defines the
coefficient $\epsilon$. This pro
multi-harmonic relative
compliance with contac

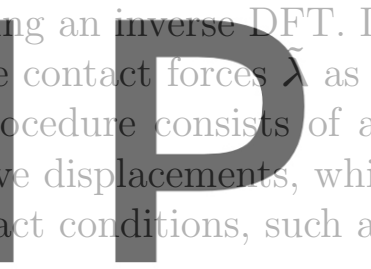

$$
\tilde{\lambda}=\tilde{\mathbb{F}}_{r}-Z_{r} \tilde{U}_{r}+\epsilon\left(\tilde{U}_{r}-\tilde{\boldsymbol{X}}_{r}\right) \text {. }
$$

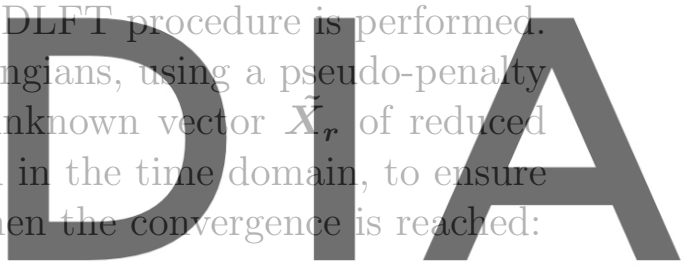

(6)

Register for free at https//www.scipedia.com to download the version without the watermark The penalty coefficient $\epsilon$ can be chosen arbitrarily since, at convergence, results do not depend on it. Charleux [3] proposed to take $\epsilon$ equals to the spectral radius of the stiffness matrix $\boldsymbol{K}$. The contact forces can be split as follows:

$$
\tilde{\boldsymbol{\lambda}}=\underbrace{\tilde{\boldsymbol{F}}_{\boldsymbol{r}}-\boldsymbol{Z}_{r} \tilde{U}_{\boldsymbol{r}}+\epsilon \tilde{\boldsymbol{U}}_{r}}_{\tilde{\boldsymbol{\lambda}}_{u}}-\underbrace{\epsilon \tilde{\boldsymbol{X}}_{r}}_{\tilde{\boldsymbol{\lambda}}_{x}},
$$

where $\tilde{\boldsymbol{\lambda}}_{\boldsymbol{u}}$ represents the term of prediction to determine the stick-slip behaviour and $\tilde{\lambda}_{x}$ is the correction term. The correction is applied by imposing its value to ensure the validation of Coulomb's friction law and unilateral contact law. Specifically, the contact forces are computed in normal and tangential direction as follows:

$$
\begin{aligned}
\tilde{\boldsymbol{\lambda}}_{N} & =\tilde{\boldsymbol{F}}_{\boldsymbol{r} N}-\left(Z_{\boldsymbol{r}} \tilde{U}_{r}\right)_{N}+\epsilon_{N}\left(\tilde{U}_{r N}-G-\tilde{\boldsymbol{X}}_{r N}\right), \\
\tilde{\boldsymbol{\lambda}}_{\boldsymbol{T}} & =\tilde{\boldsymbol{F}}_{\boldsymbol{r} \boldsymbol{T}}-\left(\boldsymbol{Z}_{\boldsymbol{r}} \tilde{\boldsymbol{U}}_{\boldsymbol{r}}\right)_{\boldsymbol{T}}+\epsilon_{T}\left(\tilde{\boldsymbol{U}}_{\boldsymbol{r} \boldsymbol{T}}-\tilde{\boldsymbol{X}}_{\boldsymbol{r} \boldsymbol{T}}\right) .
\end{aligned}
$$

The wear depth is implemented as an interface gap $G=\tilde{W}_{N}$ in normal direction as shown in Eq. (8a). The couple of unknowns $\left(\tilde{\boldsymbol{\lambda}}, \tilde{\boldsymbol{X}}_{\boldsymbol{r}}\right)$ is determined through the AFT procedure. 


\subsection{Wear computation}

Wear is computed using the Archard's model [12], over one vibration cycle. Therefore, to decrease the number of iterations and the computational time, a jump cycles strategy is implemented, assuming the wear rate to be constant for a certain number of vibration cycles, $\Delta N$, previously chosen. Denoting $\delta W^{m}$ the nodal (index $m$ ) wear rate (worn volume per unit sliding distance):

$$
\delta W^{m}(\eta)=\int_{\eta}^{\eta+T} K_{w}\left|P_{N}^{m}(\tau, \eta)\right|\left\|\dot{\boldsymbol{U}}_{\boldsymbol{T}}^{\boldsymbol{m}}(\tau, \eta)\right\| d \tau,
$$

in which $T$ is the period of the harmonic excitation force, $K_{w}$ is the Archard's wear coefficient, obtained from experimental results. $P_{N}^{m}$ and $U_{T}^{m}$ are respectively the normal load and the relative tangential velocity at each node $m$, calculated through Eq. (5).

The wear nodal depth $W^{m}$ is evaluated by integrating the following explicit scheme:

$$
W^{m}\left(\eta^{k+1}\right)=W^{m}\left(\eta^{k}\right)+\Delta N\left(\eta^{k}\right) \delta W^{m}\left(\eta^{k}\right),
$$

where $k$ represents the intermediates slow time steps. $\Delta N\left(\eta^{k}\right)$ is fixed a priori as follows:

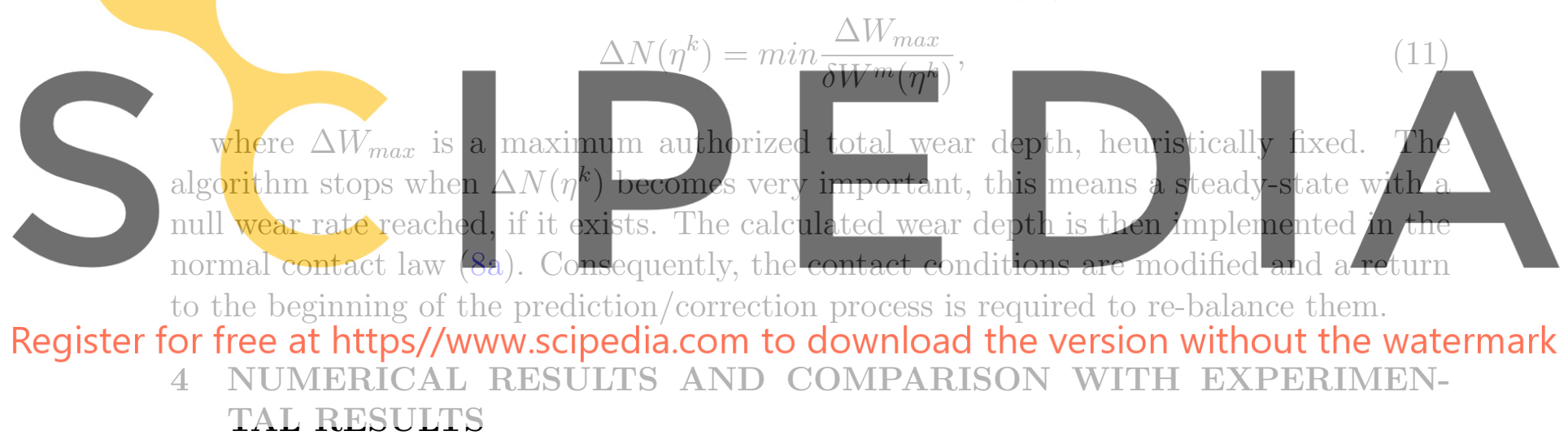

In this section, the computational method previously described in section 3 is applied to evaluate the forced response of the experimental test rig detailed in section 2. Compared to the numerical analysis performed in [10], where the contact behaviour was modelled using a Bouc-Wen element, in this paper, a FE model of the specimens has been created to study a more detailed contact behaviour which allows to analyse the status of each point inside the contact and at the end the worn profile.

\subsection{Description of the FE model}

The rig dynamics has been modelled by means of a simple 2 DOFs lumped mass model with springs to reproduce the two main sliding modes of the friction rig (the in-phase mode $\approx 40 \mathrm{~Hz}$ and the out-of-phase mode $\approx 2500 \mathrm{~Hz}$ ). The scheme of the lumped model of the rig, rigidly connected to a detailed FE model of the specimens, as shown in Figure 3a, to accurately model the contact interface conditions. The FE model of the specimens was 
created and meshed using 8-nodes quadratic brick elements. The contact area nominally flat is $1 \mathrm{~mm}^{2}$ and composed of 10x10 elements (121 contact nodes), as shown in Figure 3b. The interface mesh is compatible at each contact node.

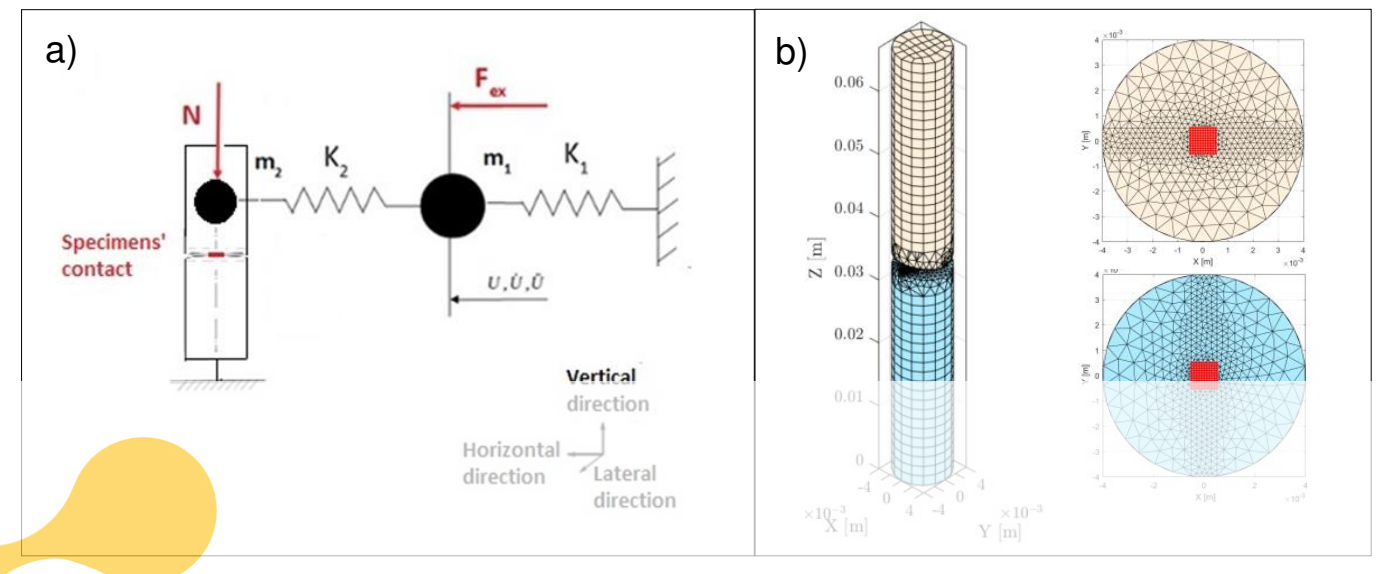

Figure 3: a) 2 DOFs lumped mass model of the rig rigidly connected to the specimens;

b) FE model of the specimens with a zoom of the contact interface
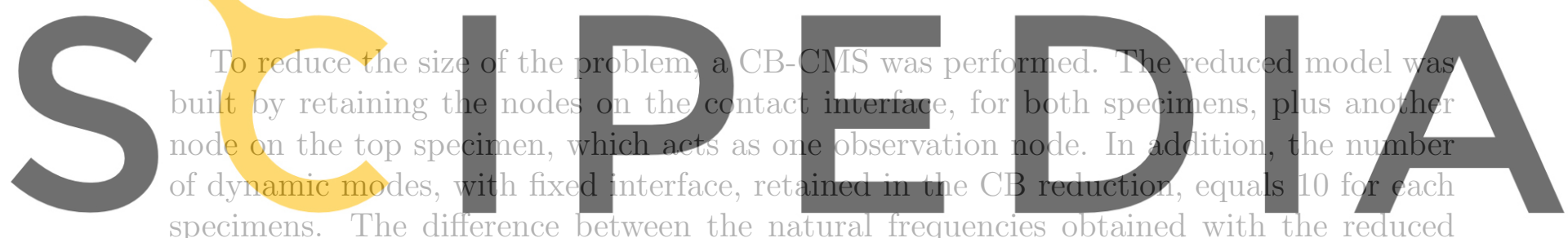

specimens. The difference between the natural frequencies obtained with the reduced

Register for free at hase ghtained for the full size model is less than $0.1 \%$. The size of the system is further reduced with a condensation procedure on the non-linear (contact) DO Fs and

then on relative displacement.

\subsection{Non-linear dynamic analysis}

The test 1 is chosen for the numerical simulations. The contact surfaces are assumed perfectly flat and smooth without any initial separation. The initial value of the gap in Eq. (8a) is $G=0$. The friction coefficient is $\mu=0.88$. This value corresponds to the steady friction coefficient reached for all the five wear tests, as defined in [10]. $K_{w}$ is calculated using the experimental data, by means of the Archard's wear formulation [12], taking into account the total wear volume. For the test $1: K_{w}=0.1110^{-7} \mathrm{~m}^{3} / \mathrm{mN}$. Concerning the HBM, 3 harmonics have been used, with 128 time steps in DFT procedure.

To perform the initial non-linear dynamic analysis, the static normal load is required. Figure 4a. displays the normal load distribution over the full interface. This repartition is the typical one expected for a flat-to-flat contact [15] with higher values exhibited on the corners of the interface, which is expected given the singularity of the stress fields at 
these locations. It has been shown a stick-slip behaviour over the interface, with stick on the middle, where $T<\neq \mu N$, and slip on the borders, where $T=\mu N$, Figure $4 \mathrm{~b}$.
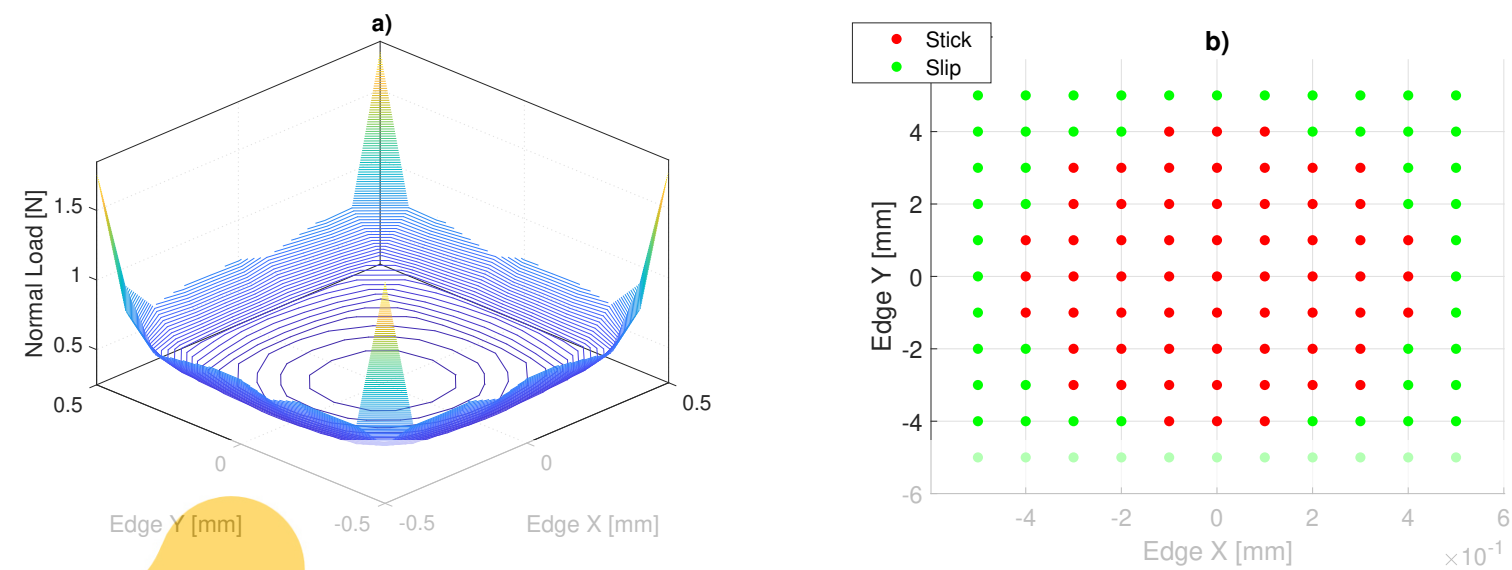

Figure 4: Contact conditions over the interface: a) Initial normal Load distribution over the interface b) Stick-slip behaviour on the contact nodes
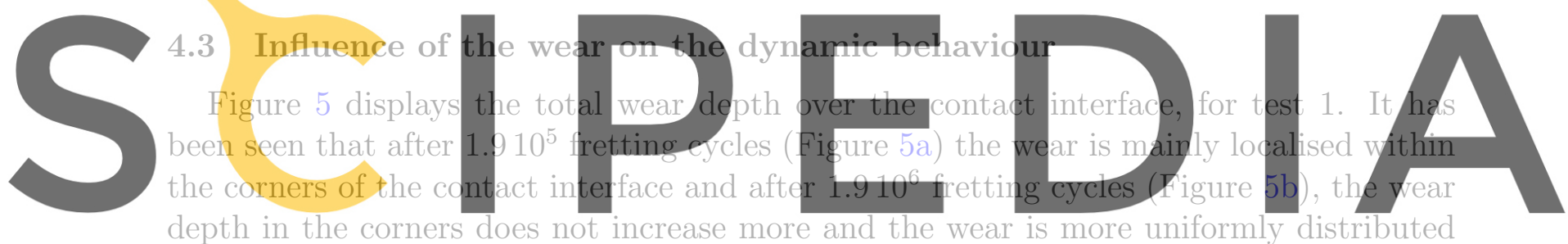

Register for free athote confact interface dia.com to download the version without the watermark
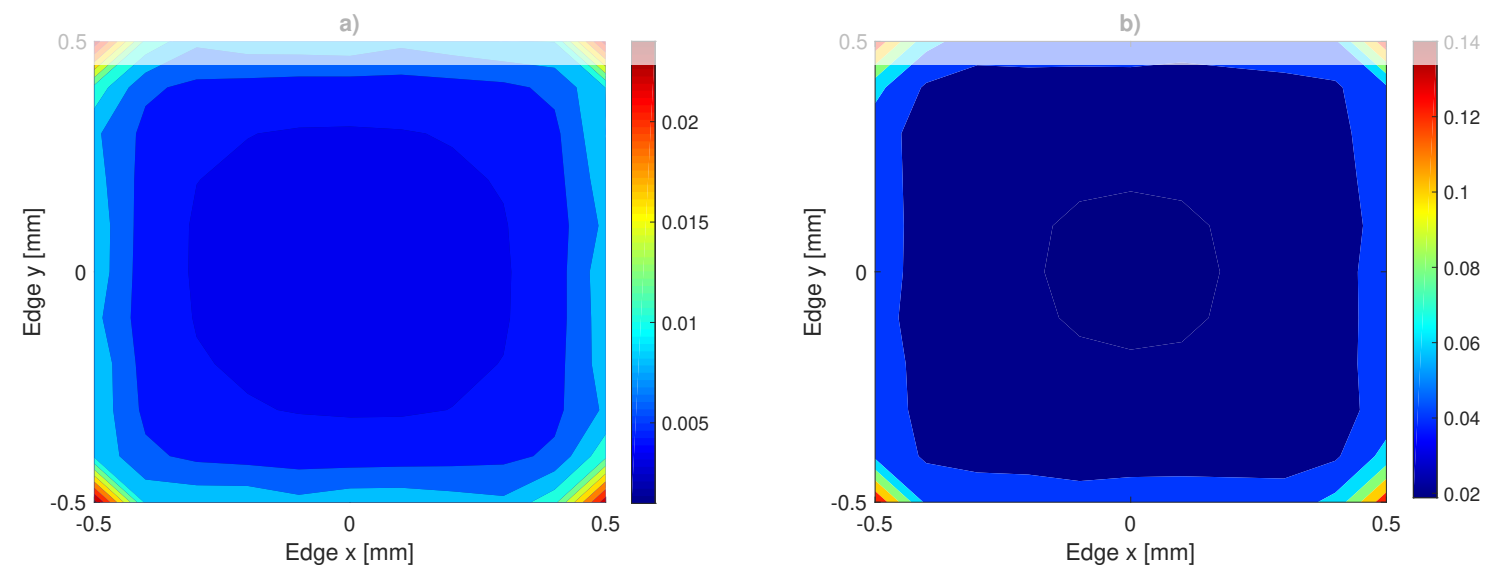

Figure 5: Wear pattern over the contact interface for the Test 1 . Wear depth in $\mu m$ : a) after $1.910^{5}$ fretting cycles and b) after $1.910^{6}$ fretting cycles 
Figure 6a displays the forced response for the unworn non-linear system and for the worn configuration after $1.910^{6}$ vibration cycles for the first mode. The numerical FRFs shows similar amplitude and resonances with the experimental ones shown in [10]. It has been observed that wear produces an increase of the first natural frequency (inphase mode), confirming that the system becomes stiffer with wear. The evolution of the numerical hysteresis loops is shown in Figure 6b, exhibiting a similar trend with the experimental ones [10].

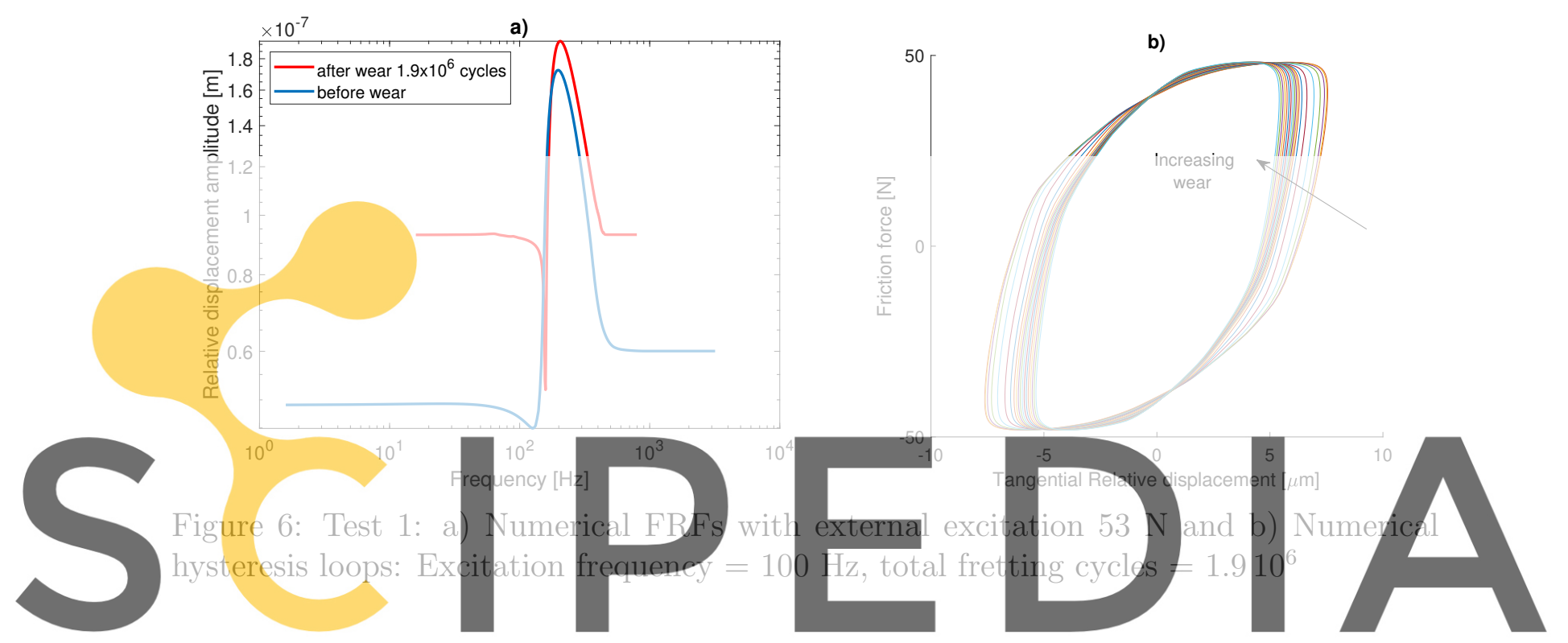

Figure 7 shows the evolution of the total worn volume for test 1. It has been observed

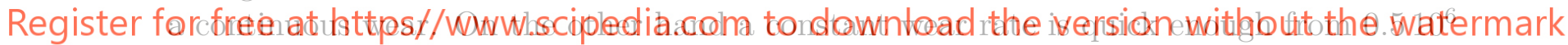
fretting cycles.

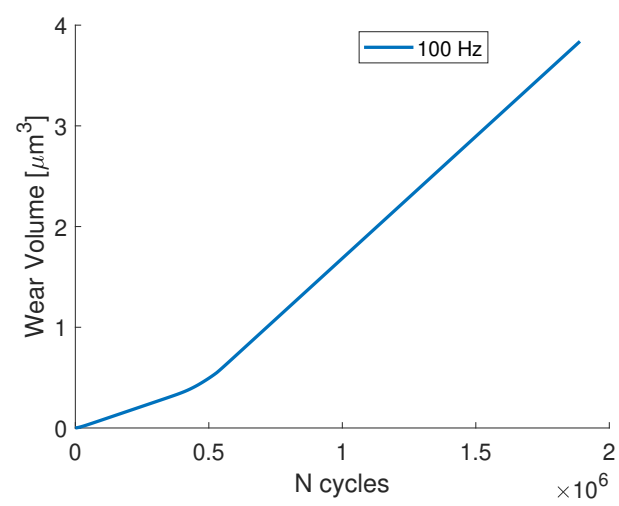

Figure 7: Evolution of the wear volume for test 1 at $100 \mathrm{~Hz}$ 


\section{CONCLUSIONS AND PROSPECTS}

The proposed numerical method based on DLFT with wear allows to calculate simultaneously the vibrations of structures and wear in fretting-wear situations by considering these two phenomena into two time scales. The jump cycles strategy, for updating wear, enables a significant reduction of the computational time. The phenomenology is quite well respected in terms of wear profile with the maximum amplitude in the corners; also total worn volume is in good agreement with experiments; moreover in term of dynamics the resonance frequency tends to increase with wear.

In terms of prospectives, the simulations have been performed for the specimens with a flat-to-flat contact area. It may be useful to consider different geometries, for instance, curved specimens and potentially more industrial cases.

The evolution of wear is here evaluated numerically. This approach allows to validate an experimental test but it does not take into account that fretting-wear generates at the interface an intermediate heterogeneous layer, composed of wear debris or particles (the so-called third body). It could be interesting in future works to include this layer. It would also be valuable to refer to an asymptotic approach to evaluate the asymptotic behaviour of the worn geometry directly without time instants integration.

\section{ACKNOWLEDGMENTS}

This project has received funding from the European Union's Horizon 2020 research and innovation programme under the Marie Sklodowska-Curie grant agreement No 721865.

\section{REFERENCES}

[1] E. P. Petrov, D. J. Ewins. Analytical Formulation of Friction Interface Elements for Analysis of Nonlinear Multi-Harmonic Vibrations of Bladed Disks, Journal of Turbomachinery, 125(2), 364, 2003. https://doi.org/10.1115/1.1539868

[2] S. Nacivet, C. Pierre, F. Thouverez and L. Jezequel, A dynamic Lagrangian frequency-time method for the vibration of dry-friction damped systems. Journal of Sound and Vibration 265, pp. 201-219, (2003). https://hal.archives-ouvertes. fr/hal-01635272

[3] Charleux D, Gibert C, Thouverez F, Dupeux J. Numerical and experimental study of friction damping blade attachments of rotating bladed disks. Int J Rotating Mach 2006. http://dx.doi.org/10.1155/IJRM/2006/71302

[4] Schwingshackl CW, Petrov EP, Ewins DJ. Measured and estimated friction interface parameters in a nonlinear dynamic analysis. In: Interdisciplinary and integration aspects in structural health monitoring. Mech Syst Signal Process (2012);28:574-84. http://dx.doi.org/10.1016/j.ymssp.2011.10.005 
[5] Salles, L., Blanc, L., Thouverez, F., Gouskov, A.M., Jean, P.: Dynamic analysis of a bladed disk with friction and fretting-wear in blade attachments. In: ASME Turbo Expo 2009: Power for Land, Sea, and Air, pp. 465-476. American Society of Mechanical Engineers, New York, (2009). https://doi.org/10.1115/GT2009-60151

[6] L. Salles, L. Blanc, F. Thouverez, A.M. Gouskov, Dynamic analysis of fretting-wear in friction contact interfaces, International Journal of Solids and Structures, Volume 48, Issue 10, 2011, Pages 1513-1524, ISSN0020-7683. https://doi.org/10.1016/j . ijsolstr.2011.01.035

[7] L. Gallego, D. Nélias, C. Jacq. A Comprehensive Method to Predict Wear and to Define the Optimum Geometry of Fretting Surfaces, Journal of Tribology, 128(3), 476-485, (2006) https://doi.org/10.1115/1.2194917.

[8] J. Armand, L. Pesaresi, L. Salles, and C. W. Schwingshackl, "A Multiscale Approach for Nonlinear Dynamic Response Predictions with Fretting Wear," J. Eng. Gas Turbines Power, vol. 139, no. 2, pp. 1-7,(2017), https://doi.org/10.1115/1.4034344.

[9] E. Lemoine, D. Nélias, F. Thouverez, C. Vincent, Influence of fretting wear on bladed disks dynamic analysis, Tribology International, Volume 145, 2020, 106148, ISSN 0301-679X, https://doi.org/10.1016/j.triboint.2019.106148.

[10] A. Fantetti, L.R. Tamatam, M. Volvert, I. Lawal, L. Liu, L. Salles, M.R.W. Brake, C.W. Schwingshackl, D.Nowell, The impact of fretting wear on structural dynamics: Experiment and Simulation, Tribology International, Volume 138, pp. 111124,(2019). https://doi.org/10.1016/j.triboint.2019.05.023

[11] A. Fantetti, C. Schwingshackl, Effect of friction on the structural dynamics of built-up structures: an experimental study, in: Proceedings of the ASME Turbo Expo 2020: Turbomachinery Technical Conference and Exposition. Volume 11, (2020).https: //doi.org/10.1115/GT2020-14945

[12] Archard JF. Contact and rubbing of flat surfaces. J Appl Phys 1953

[13] Craig R, Bampton M. Coupling of substructures for dynamic analyses. AIAA J 1968;6(7):1313-9. https://hal . archives-ouvertes.fr/hal-01537654/document

[14] Cameron TM, Griffin JH. An alternating frequency/time domain method for calculating the steady-state response of nonlinear dynamic systems. J Appl Mech 1989;56(1):149-54. http://dx.doi.org/10.1115/1.3176036.

[15] Ciavarella M, Hills DA, Monno G. The influence of rounded edges on indentation by a flat punch. Proceedings of the Institution of Mechanical Engineers, Part C: Journal of Mechanical Engineering Science. 1998;212(4):319-327.https://doi .org/ $10.1243 / 0954406981521259$. 